\title{
A study of automotive workers anthropometric physical characteristics from Mexico Northwest
}

\author{
Karla Lucero-Duarte ${ }^{\mathrm{a},{ }^{*}}$, Enrique de la Vega-Bustillos ${ }^{\mathrm{b}}$, Francisco López-Millán ${ }^{\mathrm{a}}$ \\ ${ }^{a}$ Departamento de ingeniería Industrial, Instituto Tecnológico de Hermosillo, Av. Tecnológico S/N, Hermosillo, \\ Sonora, México \\ ${ }^{\mathrm{b}}$ División de Estudios de Posgrado e Investigación, Instituto Tecnológico de Hermosillo, Av. Tecnológico S/N, \\ Hermosillo, Sonora, México
}

\begin{abstract}
Due to the lack of anthropometric information in northwest Mexico, we did an anthropometric study that represents the population physical characteristics and that is reliable for the design or redesign of workstations. The study was divided in two phases. The first one was the anthropometric study of 2900 automotive industry workers in northwest of Mexico. The study includes 40 body dimensions of 2345 males and 555 females personalized to be used in future researches. Second phase includes compared anthropometric characteristics of population reported in four Mexican studies and a Colombian study against the current study. Benefits of this project are: a reliable database of anthropometric characteristic of automotive industry population for workstations design or redesign that match with the users, increase product quality and reduce economic, medical and union complains.
\end{abstract}

Keywords: Work design; workstation design; populations comparison

\section{Introduction}

The main tool for designers of workplaces is anthropometric data which provide information on critical body dimension, unfortunately, in Mexico there are a few anthropometric studies and are too regionalized, the sample size is too small and only provide information for men or women. This is the case in Avila et al [1] presented a study at Guadalajara, Mexico metropolitan area. Lavander et al [2] presents a study of 21 anthropometric measures on 87 female workers in maquiladora industry in the Mexico - U.S.A. border. Liu et al [3] presents a study of only 12 anthropometric measures on 110 female workers in the maquiladora industry in the Mexico - U.S.A. border. These last two studies were conducted in Mexico northeast zone.

This paper presents results of 40 anthropometric measurements of automotive industry workers, males and females. These data are compared with anthropometric characteristics of some population reported in Guadalajara, Leon Guanajuato, Mexico City and Colombia.

\section{Methods}

\subsection{Subjects}

The mean age and standard deviation were 30.24 and 7.04 year for female and 33.51 and 8.19 year for male respectively. The mean and standard deviation of the weight of the participants were 81.21 Kg and $12.24 \mathrm{Kg}$ for male and $69.52 \mathrm{Kg}$ and $11.73 \mathrm{Kg}$ for female res respectively. The sample presented in the present study seems to be representative of the employees of the automotive industry at northwest of Mexico

*Corresponding author. E-mail: kalucero00@hotmail.com 


\subsection{Apparatus}

Standard tools for anthropometric measurement, including calipers and anthropometers, were used for length and breadth measurements of various body segments. A graduated cone was used to measure the grip diameter of the dominant hand. Maximum grip strength was measured using a hand dynamometer with the handle width set to $5 \mathrm{~cm}$. Weight was measured using a physician's scale. Circumferences were measured with a plastic tape graduated in millimeters.

\subsection{Procedures}

2,345 male and 555 female operators were selected to participate in the survey. All were employees at an automotive industry facility in Mexico northwest. Thus, sample was limited to a regional population segment. All employees were measured wearing work clothes and safety shoes, where measurements were made on the right side of the body. Data were obtained for each of the measures defined in Table I. Where dimensions measured were consistent with those summarized by NASA [4]. Test statistics were conducted with Minitab®.

\section{Results}

Table 1 summarizes the anthropometric characteristics of the sample by gender. The mean and standard deviation of stature from this sample were $173.47 \mathrm{~cm}$ and $6.52 \mathrm{~cm}$ for male and $163.29 \mathrm{~cm}$ and $5.87 \mathrm{~cm}$ for female respectively with shoes on. Table 2 also provides the values of each body dimension at the 5th, 50th and 95th percentile.

\subsection{Comparison with other populations}

By making a comparison of data from current study with data from other populations, with the subject standing, it shows that there is a significant difference among data. Tables 2 and Table 3 show the data used to make comparisons between different populations

\section{Discussion}

This paper presents 40 measures anthropometric essentials for the design of workstations, tools, clothing or personal protective equipment. Based on the results of the comparisons with other studies with populations of Mexico, we can conclude that workers in northwestern Mexico are higher and the fact that measures have been taken with no shoes affects the comparison with other studies. Finally, the range and average age of this study, 18 to 61 years and 32.89 years is similar to other studies presented here.

\section{References}

[1] Ávila, R., Prado, L. González, E. Dimensiones antropométricas. Población Latinoamericana. Ed. Universidad de Guadalajara. 2007, pp. 94-123

[2] Lavander S., Marras W. and Sabol S.. A study of female mexican anthropometric measures useful for workstation design in light manufacturing facilities. AIHA Journal, ed. Taylor \& Francis, 2002, pp. 300-304

[3] Liu, W.C., D. Sanchez-Monrroy, and G. Parga (1999). Anthropometry of female maquiladora workers. International Journal of Industrial Ergonomics.ed. Elsevier 1999, pp. 273 280

[4] NASA (National Aeronautics and Space Administration), Anthropology Research Project 1978 Anthropometric Source Book, Vol. I: Anthropometry for Designers, NASA. Reference Publication 1024' Webb Associates (Ed.). National Aeronautics and Space Administration Scientific and Technical Information Office, Houston, Texas, USA

Table 1

Anthropometric characteristics

\begin{tabular}{|c|c|c|c|c|c|c|c|c|}
\hline & \multicolumn{4}{|c|}{ MALE } & \multicolumn{4}{|c|}{ FEMALE } \\
\hline & average & std dv & $5 \%$ & $95 \%$ & average & std dv & $5 \%$ & $95 \%$ \\
\hline Stature & 173.47 & 6.52 & 162.84 & 184.00 & 163.29 & 5.87 & 153.94 & 172.50 \\
\hline Eye height & 162.58 & 6.50 & 152.50 & 173.30 & 151.86 & 5.68 & 142.50 & 160.50 \\
\hline Shoulder height & 144.75 & 6.39 & 135.70 & 154.10 & 135.79 & 5.21 & 127.57 & 144.06 \\
\hline Elbow height & 111.69 & 4.80 & 104.30 & 119.50 & 105.23 & 4.97 & 98.50 & 112.40 \\
\hline Waist height & 104.29 & 5.84 & 95.00 & 114.00 & 99.01 & 5.77 & 89.50 & 108.23 \\
\hline Buttocks height & 78.50 & 4.39 & 71.50 & 85.60 & 74.46 & 3.93 & 68.04 & 80.83 \\
\hline $\begin{array}{l}\text { Height to the } \\
\text { wrist }\end{array}$ & 86.20 & 4.49 & 79.60 & 93.30 & 81.94 & 4.12 & 75.27 & 88.23 \\
\hline $\begin{array}{l}\text { Middle finger } \\
\text { height }\end{array}$ & 67.57 & 3.76 & 61.70 & 73.70 & 64.66 & 3.47 & 59.57 & 70.13 \\
\hline Span & 176.46 & 7.13 & 164.70 & 188.48 & 161.89 & 6.50 & 151.57 & 172.00 \\
\hline $\begin{array}{l}\text { Elbows width } \\
\text { with the hands } \\
\text { on the center } \\
\text { chest. }\end{array}$ & 82.68 & 7.86 & 71.20 & 94.60 & 75.40 & 7.24 & 64.77 & 86.30 \\
\hline $\begin{array}{l}\text { Arm reach from } \\
\text { wall }\end{array}$ & 87.60 & 4.26 & 80.72 & 94.68 & 81.05 & 3.93 & 75.14 & 87.60 \\
\hline
\end{tabular}


Table 1. Continuation

\begin{tabular}{|c|c|c|c|c|c|c|c|c|}
\hline & \multicolumn{4}{|c|}{ MALE } & \multicolumn{4}{|c|}{ FEMALE } \\
\hline & average & std dv & $5 \%$ & $95 \%$ & average & std dv & $5 \%$ & $95 \%$ \\
\hline $\begin{array}{l}\text { Arm reach from } \\
\text { wall with closed } \\
\text { hand }\end{array}$ & 77.96 & 4.05 & 71.60 & 84.60 & 72.11 & 3.80 & 66.10 & 78.10 \\
\hline $\begin{array}{l}\text { Chest } \\
\text { circumference }\end{array}$ & 102.76 & 8.35 & 90.00 & 117.16 & 96.49 & 7.13 & 86.00 & 108.00 \\
\hline $\begin{array}{l}\text { Waist } \\
\text { circumference }\end{array}$ & 96.05 & 9.65 & 81.00 & 112.96 & 88.99 & 11.09 & 72.00 & 109.00 \\
\hline $\begin{array}{l}\text { Buttock } \\
\text { circumference }\end{array}$ & 103.56 & 6.75 & 93.00 & 115.00 & 104.96 & 8.34 & 93.00 & 120.00 \\
\hline Hand length & 18.82 & 0.88 & 17.40 & 20.20 & 17.48 & 0.79 & 16.20 & 18.80 \\
\hline Palm length & 10.77 & 0.66 & 9.80 & 11.70 & 10.00 & 0.56 & 9.10 & 11.00 \\
\hline Hand breadth & 8.79 & 0.50 & 8.00 & 9.60 & 7.81 & 0.51 & 7.10 & 8.70 \\
\hline Grip diameter & 48.11 & 3.87 & 42.00 & 54.00 & 45.62 & 3.63 & 40.00 & 52.00 \\
\hline Sitting height & 89.58 & 3.21 & 84.30 & 94.80 & 85.26 & 3.08 & 80.07 & 90.23 \\
\hline Eye height & 78.77 & 3.65 & 72.80 & 84.70 & 73.92 & 3.21 & 68.94 & 78.90 \\
\hline Shoulder height & 61.27 & 3.32 & 56.20 & 66.58 & 58.27 & 2.84 & 54.00 & 63.06 \\
\hline Elbow 1 & 26.55 & 2.96 & 21.62 & 31.20 & 26.77 & 2.77 & 22.10 & 31.30 \\
\hline $\begin{array}{l}\text { Thigh clearance } \\
\text { height }\end{array}$ & 15.09 & 1.79 & 12.40 & 18.10 & 14.76 & 2.10 & 11.50 & 18.20 \\
\hline $\begin{array}{l}\text { Vertical arm } \\
\text { reach from seat }\end{array}$ & 134.21 & 5.32 & 125.60 & 143.00 & 124.96 & 4.49 & 117.47 & 132.30 \\
\hline
\end{tabular}

Table 1.Continuation

\begin{tabular}{|c|c|c|c|c|c|c|c|c|}
\hline & \multicolumn{4}{|c|}{ MALE } & \multicolumn{4}{|c|}{ FEMALE } \\
\hline & average & std dv & $5 \%$ & $95 \%$ & average & std dv & $5 \%$ & $95 \%$ \\
\hline $\begin{array}{l}\text { Vertical arm } \\
\text { reach from seat } \\
\text { with closed } \\
\text { hand }\end{array}$ & 124.20 & 5.07 & 115.92 & 132.48 & 115.84 & 4.29 & 108.87 & 122.80 \\
\hline Sitting height & 132.28 & 4.33 & 125.30 & 139.50 & 125.77 & 4.43 & 118.20 & 132.63 \\
\hline Seat height & 42.32 & 2.40 & 38.40 & 46.28 & 40.37 & 2.69 & 36.00 & 44.50 \\
\hline $\begin{array}{l}\text { Buttock to } \\
\text { popliteal length } \\
\text { seated }\end{array}$ & 47.94 & 3.23 & 43.00 & 53.20 & 47.34 & 3.25 & 42.50 & 52.46 \\
\hline $\begin{array}{l}\text { Buttock knee } \\
\text { length seated }\end{array}$ & 59.41 & 3.61 & 53.82 & 65.10 & 57.60 & 3.51 & 52.07 & 63.43 \\
\hline Popliteal height & 44.31 & 3.10 & 39.60 & 49.78 & 42.68 & 2.74 & 38.47 & 46.76 \\
\hline Knee height & 53.74 & 3.10 & 48.82 & 58.88 & 51.31 & 2.89 & 47.00 & 55.73 \\
\hline $\begin{array}{l}\text { Forearm and } \\
\text { hand length }\end{array}$ & 46.96 & 2.21 & 43.50 & 50.50 & 43.13 & 2.39 & 40.10 & 46.40 \\
\hline $\begin{array}{l}\text { Shoulders } \\
\text { breadth }\end{array}$ & 43.77 & 2.71 & 39.50 & 48.30 & 39.31 & 2.30 & 35.70 & 43.50 \\
\hline Hips breadth & 36.77 & 2.84 & 32.40 & 41.40 & 39.04 & 3.61 & 33.60 & 45.00 \\
\hline Thigh breadth & 32.82 & 3.24 & 27.90 & 38.10 & 35.10 & 4.37 & 28.27 & 42.10 \\
\hline $\begin{array}{l}\text { Vertical arm } \\
\text { reach with } \\
\text { closed hand }\end{array}$ & 214.77 & 9.80 & 199.12 & 232.00 & \begin{tabular}{|l|}
196.66 \\
\end{tabular} & 8.36 & 184.00 & 211.00 \\
\hline $\begin{array}{l}\text { Vertical arm } \\
\text { reach }\end{array}$ & 110.49 & 10.17 & 94.80 & 126.00 & 97.68 & 8.18 & 86.57 & 112.30 \\
\hline Hand force & 46.03 & 7.88 & 32.00 & 58.04 & 27.50 & 8.16 & 12.00 & 39.00 \\
\hline
\end{tabular}

Table 2. Comparison among male in the current study with other populations

\begin{tabular}{|c|c|c|c|c|c|c|c|c|c|c|}
\hline \multirow[b]{2}{*}{ Dimension } & & \multicolumn{5}{|c|}{ STANDING } & \multicolumn{4}{|c|}{ SITTING } \\
\hline & & $\begin{array}{c}\text { current study } \\
\mathrm{n}=2,345\end{array}$ & $\begin{array}{c}\text { Colombia } \\
\text { Estrada } \mathrm{n}=487\end{array}$ & $\begin{array}{l}\text { Guadalajara } \\
\text { Avila n=396 }\end{array}$ & $\begin{array}{c}\text { Leon Avila } \\
\mathrm{n}=509\end{array}$ & $\begin{array}{l}\text { Mexico city, } \\
\text { Avila n }=974\end{array}$ & $\begin{array}{c}\text { current study } \\
n=2,345\end{array}$ & $\begin{array}{c}\text { Colombia } \\
\text { Estrada } \mathrm{n}=487\end{array}$ & $\begin{array}{l}\text { Guadalajara } \\
\text { Avila n=396 }\end{array}$ & $\begin{array}{c}\text { Leon } \\
\text { Avila n }=509\end{array}$ \\
\hline \multirow[t]{2}{*}{ stature } & Media & 173.47 & 170.1 & 167.5 & 164.37 & 164.758 & 89.58 & 89 & 87.6 & 85.59 \\
\hline & SE & 6.52 & 6.52 & 6.28 & 6.03 & 5.644 & 3.21 & 3.31 & 3.117 & 1.22 \\
\hline \multirow[t]{2}{*}{ Eye height } & Media & 162.58 & 159.1 & 155 & 152.73 & 154.75 & 78.77 & 78.6 & & 74 \\
\hline & SE & 6.5 & 6.36 & 6.18 & 5.91 & 5.873 & 3.65 & 3.31 & & 3.17 \\
\hline Shoulder & Media & 144.75 & 138.6 & 138 & 135.92 & 137.269 & 61.27 & 58.8 & 58.1 & 57.15 \\
\hline Height & SE & 6.39 & 5.72 & 5.849 & 5.52 & 5.634 & 3.32 & 2.78 & 2.763 & 2.85 \\
\hline Elbow & Media & 111.69 & 107 & 106.8 & 101.06 & 103.119 & 26.55 & 23.6 & 24.6 & 22.22 \\
\hline Height & SE & 4.8 & 8.7 & 5.502 & 4.59 & 4.951 & 2.96 & 2.42 & 2.836 & 3.02 \\
\hline
\end{tabular}

\begin{tabular}{|c|c|c|c|c|c|c|c|c|c|}
\hline \multirow[b]{2}{*}{ Dimension } & & \multicolumn{5}{|c|}{ STANDING } & \multicolumn{3}{|c|}{ SITTING } \\
\hline & & $\begin{array}{c}\text { Current Study } \\
n=555\end{array}$ & $\begin{array}{c}\text { Colombia, } \\
\text { Estrada } n=233\end{array}$ & $\begin{array}{l}\text { Guadalajara, } \\
\text { Avila n }=204\end{array}$ & Lavander $n=87$ & Liv $n=110$ & \begin{tabular}{|c|}
$\begin{array}{c}\text { Current Study } \\
\mathrm{n}=555\end{array}$ \\
\end{tabular} & $\begin{array}{c}\text { Colombia, } \\
\text { Estrada } n=233\end{array}$ & $\begin{array}{l}\text { Guadalajara, } \\
\text { Avila n=204 }\end{array}$ \\
\hline stature & Media & 163.29 & 156.9 & 156.7 & 156.3 & 153.5 & 85.26 & 83.7 & 83.2 \\
\hline & SE & 5.87 & 5.8 & 5.292 & 5.2 & 5.5 & 3.08 & 2.8 & 2.742 \\
\hline Eye height & Media & 151.86 & 146.3 & 144.9 & 145.1 & 142.8 & 73.92 & 73.4 & \\
\hline & SE & 5.68 & 5.665 & 5.242 & 4.9 & 5.6 & 3.21 & 2.82 & \\
\hline Shoulder & Media & 135.79 & 128 & 129.1 & 129.5 & & 58.27 & 55.4 & 55.1 \\
\hline Height & SE & 5.21 & 5.05 & 4.917 & 4.7 & & 2.84 & 2.3 & 2.295 \\
\hline & Media & 105.23 & 98.9 & 100.4 & 97.2 & 95.6 & 26.77 & 23.3 & 25 \\
\hline Elbow & & & & & & & & & \\
\hline Height & SE & 4.97 & 4.04 & 3.889 & 4.1 & 3.8 & 2.77 & 2.06 & 1.52 \\
\hline
\end{tabular}

